\title{
PRODUÇÃO DE SERAPILHEIRA E CICLAGEM DE NUTRIENTES DE UMA FLORESTA ESTACIONAL SEMIDECIDUAL EM ZONA RIPÁRIA ${ }^{1}$
}

Ana Rosa Tundis Vital ${ }^{2}$, Iraê Amaral Guerrini ${ }^{3}$, Wolfram Karl Franken ${ }^{4}$ e Renata Cristina Batista Fonseca ${ }^{5}$

\begin{abstract}
RESUMO - O presente trabalho foi realizado em uma zona ripária no período de outubro de 2000 a setembro de 2001, em uma parcela representativa de mata ciliar com vegetação do tipo "Floresta Estacional Semidecidual", localizada no centro-sul do Estado de São Paulo. A produção total de serapilheira foi de $10.646,0 \mathrm{~kg} \cdot \mathrm{ha}^{-1} \cdot \mathrm{a}^{-1}$. A maior deposição de serapilheira e nutrientes ocorreu no fim da estação seca. A transferência total de macronutrientes foi de 217,76 kg.ha- de N, 11,55 kg.ha-1 de P, 52,79 kg.ha-1 de K, 199,80 kg.ha-1 de Ca e 38,70 kg.ha-1 de Mg. A serapilheira acumulada foi estimada em $6.227,25 \mathrm{~kg} \cdot \mathrm{ha}^{-1}$, a estimativa da taxa instantânea de decomposição (K), de 1,71; e o tempo necessário para o desaparecimento de 50 e $95 \%$ da serapilheira produzida, 150 e 639 dias, respectivamente.
\end{abstract}

Palavras-chave: Mata ciliar, floresta estacional semidecidual, serapilheira e nutrientes.

\section{LITTER PRODUCTION AND NUTRIENT CYCLING OF A SEMIDECIDUOUS MESOPHYTIC FOREST IN A RIPARIAN ZONE}

\begin{abstract}
This work was carried out in a riparian zone from October 2000 to September 2001, within a representative plot of a riparian forest with semideciduous mesophytic forest vegetation, located in the

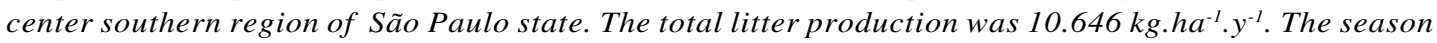
of highest litter and nutrient deposition was the late dry season. The total macronutrient transfer was 217.76 kg.ha ${ }^{-1} \mathrm{~N} ; 11.55 \mathrm{~kg} . \mathrm{ha}^{-1} \mathrm{P} ; 52.79 \mathrm{~kg} . \mathrm{ha}^{-1} \mathrm{~K} ; 199.80 \mathrm{~kg} . \mathrm{ha}^{-1} \mathrm{Ca}$ and $38.70 \mathrm{~kg} . \mathrm{ha}^{-1} \mathrm{Mg}$. The accumulated litter was estimated as $6.227 \mathrm{~kg} \mathrm{ha}^{-1}$, instantaneous decomposition rate (K) 1.71 and time needed time for $50 \%$ and $95 \%$ litter disappearance was 150 and 639 days, respectively.
\end{abstract}

Key words:Riparian forest, nutrient cycling, litter, nutrient, decomposition.

\section{INTRODUÇÃO}

O estudo da ciclagem de nutrientes minerais, via serapilheira, é fundamental para o conhecimento da estrutura e funcionamento de ecossistemas florestais. Parte do processo de retorno de matéria orgânica e de nutrientes para o solo florestal se dá através da produção de serapilheira, sendo esta considerada o meio mais importante de transferência de elementos essenciais da vegetação para o solo. Em zonas de troca entre os ecossistemas terrestres e aquáticos chamados de zonas ripárias existem alguns processos de transferência importantes, exclusivos de matas ciliares: o primeiro é a entrada de sedimentos a partir das áreas adjacentes, transportados pelas águas das chuvas

\footnotetext{
${ }^{1}$ Recebido em 29.04.2003 e aceito para publicação em 10.08.2004.

${ }^{2}$ Instituto Nacional de Pesquisas da Amazônia - INPA/MCT.

${ }^{3}$ Departamento de Recursos Naturais e Ciência do Solo, FCA/UNESP, Campus de Botucatu, Botucatu, SP.

${ }^{4}$ Coordenação de Pesquisas em Clima e Recursos Hídricos, Instituto Nacional de Pesquisas da Amazônia - INPA/MCT.

${ }^{5}$ Departamento de Recursos Naturais/Ciências Florestais, FCA/UNESP, Campus de Botucatu, Botucatu, SP.
} 
ou de rio, sendo retidos pela faixa florestal que atua como filtro; o segundo trata da entrada de nutrientes também através do fluxo lateral do lençol freático, transportando nutrientes das partes mais elevadas para a faixa ciliar (PAGANO e DURIGAN, 2000). Os fatores que afetam a forma e a ciclagem de nutrientes estão intimamente ligados às condições climáticas e fenológicas, bem como aos aspectos ambientais e aos poluentes, variando de espécie para espécie (SCHUMACHER, 1992; POGGIANI e SCHUMACHER, 2000). Em geral, observa-se um aumento da deposição da serapilheira até a idade em que as árvores atingem a maturidade ou fecham as suas copas. Após esse ponto podem ocorrer ligeiro decréscimo ou estabilização (BRAY e GHORAN, 1964).

Não existe informação detalhada sobre a ciclagem de nutrientes em florestas tropicais e, menos ainda, especificamente em matas ciliares. De acordo com Pagano e Durigan (2000), especialmente em matas ciliares situadas no Estado de São Paulo, as informações são insuficientes e se restringem a poucos trabalhos. Em experimentos realizados por esses autores em cinco ambientes distintos de mata ciliar, da região oeste paulista, concluiu-se que a produção de serapilheira tem apresentado dois padrões bem distintos: (1) onde os maiores picos ocorrem no inverno, caracterizado por baixa precipitação (Floresta Estacional Semidecidual, Mata ciliar, Cerrado), (2) onde os maiores picos ocorrem na época chuvosa e de temperatura mais elevada (Floresta Atlântica), onde se encontram produções de serapilheira, em $\left(\mathrm{kg} \cdot \mathrm{ha}^{-1}\right)$ : $6.398 ; 9.744$ e 11.126 em solos secos e 5.348 e 8827 em solos molhados, respectivamente.

Em Floresta de Altitude e Floresta Estacional Semidecidual no interior do Estado de São Paulo, Morellato (1992) observou que a maior produção de serapilheira ocorre no período seco, em que encontrou uma produção de serapilheira de 7 e 8,6 t.ha-1, respectivamente. Porém, Cunha et al. (1993) e Cunha (1997), em estudo sobre a dinâmica de nutrientes em Floresta Estacional Decidual, em áreas distintas no Estado do Rio Grande do Sul, observaram que a estação de maior deposição de serapilheira e nutrientes foi a primavera, quando as precipitações foram abundantes e a temperatura média mensal estava em elevação. O primeiro autor encontrou uma produção anual de serapilheira de 7,76 t.ha ${ }^{-1}$, com deposição anual no solo florestal de 206 kg.ha $a^{-1} \cdot a^{-1}$ de N, 11,2 kg.ha ${ }^{-1} \cdot a^{-1}$ de P, 37,8 kg.ha $a^{-1} \cdot a^{-1}$ de K, 269,2 kg.ha ${ }^{-1} \cdot a^{-1}$ de Ca e 29,9 kg.ha ${ }^{-1} \cdot a^{-1}$ de Mg. A taxa de decomposição da serapilheira acumulada foi igual a 1,16 e o tempo necessário para o desaparecimento de $50 \%$ da serapilheira, igual a sete meses. Os segundos autores estimaram uma produção média de serapilheira de 5,8 th.ha $a^{-1} \cdot a^{-1}, 8,0$ t.ha $a^{-1} \cdot a^{-1}$ e 9,5 t.ha $a^{-1} \cdot a^{-1}$, respectivamente, em capoeira, capoeirão e mata. Em um fragmento florestal do tipo Floresta Estacional Semidecidual Submontana em Piracicaba, SP, Oliveira (1997) encontrou uma produção de serapilheira de $14.715,97 \mathrm{~kg} \cdot \mathrm{ha}^{-1} \cdot \mathrm{a}^{-1}$, com retorno de macronutrientes na serapilheira na seguinte ordem: $\mathrm{Ca}>\mathrm{N}>\mathrm{K}>\mathrm{Mg}>\mathrm{P}$.

A acumulação de serapilheira é variável de acordo com o ecossistema considerado e seu estádio sucessional (DELITTI, 1989). Em se tratando de mata ciliar, essa acumulação está relacionada ao teor de umidade e à fertilidade do solo que a suporta, apresentando resultados diferentes mesmo estando essas matas situadas muito próximas entre si e exibindo as mesmas condições climáticas (PAGANO e DURIGAN, 2000). As taxas de decomposição da serapilheira são consideradas rápidas, se existe pouco acúmulo na superfície do solo e os coeficientes de decomposição na condição de equilíbrio dinâmico $(\mathrm{K})$ são maiores que 1,0 (WARING e SCHLESINGER, 1985). Aconversão dessa matéria orgânica para húmus ocorre a uma taxa de 30 a $50 \%$ ao ano (GOLLEY, 1983).

O presente trabalho teve como objetivo o estudo da ciclagem de nutrientes em um ecossistema de Mata Ciliar, do tipo Floresta Estacional Semidecidual, na região centro-sul do Estado de São Paulo, sendo quantificados parâmetros quanto à produção, taxa de decomposição e caracterização química da matéria orgânica.

\section{MATERIAL E MÉTODOS}

\subsection{Localização e vegetação}

O experimento foi desenvolvido na Fazenda Experimental Edgardia, pertencente à Faculdade de Ciências Agronômicas da UNESP, Campus de Botucatu, Estado de São Paulo. Essa fazenda dispõe de área de aproximadamente 1.200 ha. Possui altitude que varia de 475 a 725 m (CARVALHO et al., 1991). A área experimental foi implantada em um trecho de Floresta Estacional Semidecidual (IBGE, 1992), chamada de Mata do Bexiguento, com aproximadamente 303 ha, situada nas coordenadas de $22^{\circ} 48^{\prime} 51^{\prime \prime} \mathrm{S}$ e $48^{\circ} 24^{\prime} 15^{\prime \prime} \mathrm{W}$ de 
Greenwich. O solo da parcela estudada foi do tipo Neossolo Litólico próximo ao Neossolo Flúvico (solos aluviais) (EMBRAPA, 1999).

De acordo com levantamento feito "in loco", o ecossistema é caracterizado pela presença de Acacia polyphylla DC., Alchornea triplinervia (Spreng.) Müll. Arg., Aspidosperma polyneuron Müll. Arg., Bastardiopsis densiflora (Hook. \& Arn.) Hassl., Cordia trichotoma (Vell.) Arrab. ex. Steud., Gallesia integrifolia (Spreng.) Harms, Luehea divaricata Mart., Machaerium brasiliense Vogel., Machaerium stipitatum (DC.) Vogel., Maytenus aquifolium Mart., Parapiptadenia rigida (Benth.) Brenan, Peltophorum dubium (Spreng.) Taub. e Trichilia elegans A. Juss., entre as mais freqüentes. A densidade populacional pode ser considerada alta, com cerca de 7.676 árvores por hectare com DAP maior que $5 \mathrm{~cm}$; as árvores dominantes na área possuíam altura máxima de $22 \mathrm{~m}$.

\subsection{Variáveis estudadas}

Para quantificar a produção de serapilheira foram utilizados quatro coletores de $1 \mathrm{~m}^{2}$ de superfície, altura de $10 \mathrm{~cm}$ de bordadura, com fundo de tela de náilon com malha de $1 \mathrm{~mm}$, suspensos a $30 \mathrm{~cm}$ do solo. Esses coletores foram instalados individualmente e distribuídos de forma casualizada em parcelas de 100 $\mathrm{m}^{2}$. Para estimar a quantidade de serapilheira produzida foram realizadas 12 coletas, com intervalo de 30 dias, no ano experimental. A serapilheira acumulada em cada um dos coletores de $1 \mathrm{~m}^{2}$ foi recolhida, pesada e embalada em sacos plásticos numerados e secada em sacos de papel em estufa à temperatura média de $60{ }^{\circ} \mathrm{C}$, pesando-os após a referida secagem em balança analítica (precisão de 0,01 g). A partir desses dados, puderam-se estimar as médias mensal e anual da serapilheira produzida.

\subsection{Análise química e quantificação de nutrientes na serapilheira}

Depois de pesado, o material da serapilheira foi moído em moinho tipo Wiley, sendo o pó resultante levado novamente à estufa e secado a $50^{\circ} \mathrm{C}$, para posterior análise química em laboratório dos macronutrientes: nitrogênio $(\mathrm{N})$, fósforo $(\mathrm{P})$, potássio $(\mathrm{K})$, cálcio $(\mathrm{Ca})$ e magnésio $(\mathrm{Mg})$.

\subsection{Eficiência de utilização de nutrientes}

A eficiência de utilização de nutrientes foi calculada para os elementos N, P, K, Ca e Mg da serapilheira, estimada através da relação entre a biomas- sa de serapilheira produzida e a quantidade de nutrientes transferidos por essa biomassa, como proposto por Vitousek (1982).

\subsection{Ciclagem de nutrientes}

A serapilheira acumulada sobre o solo foi estimada em coletas bimensais, totalizando seis coletas no decorrer do período experimental, quando foi coletada uma amostra por parcela, utilizando-se um quadrado de madeira de $0,25 \mathrm{~m}^{2}$. Para obtenção dessas amostras, foi coletada toda a manta existente sobre o solo, secando-se o material coletado em estufa a uma temperatura de $60^{\circ} \mathrm{C}$.

A taxa de decomposição da serrapilheira foi estimada a partir da equação proposta por Olson (1963):

$$
\frac{L}{X S S}=K
$$

Na equação (1), com valores dados em $\left(\mathrm{g} / \mathrm{m}^{2}\right), \mathrm{L}$ representa a quantidade de serrapilheira produzida anualmente, Xss a média anual de serrapilheira acumulada sobre o solo e $\mathrm{K}$ a constante de decomposição na condição de equilíbrio dinâmico.

Calculou-se também, a partir do valor K, o tempo médio de renovação, estimado por $1 / \mathrm{K}$. Foram calculados ainda os tempos necessários para o desaparecimento de 50 e $95 \%$ da serapilheira, segundo Shanks e Olson (1961):

$$
\begin{aligned}
& \text { T } 0,5=\mathrm{e}^{-\mathrm{kt}}{ }_{0,5} \text { ou t } 0,5=0,693 / \mathrm{K} \\
& \text { T } 0,05=3 / \mathrm{K}
\end{aligned}
$$

\section{RESULTADOS E DISCUSSÃO}

\subsection{Produção de serapilheira}

A produção de serapilheira foi estimada em 10.646,1 $\mathrm{kg} \cdot \mathrm{ha}^{-1} \cdot \mathrm{a}^{-1}$ (Figura 1).

A deposição de biomassa alcançou seu valor máximo em setembro, no fim do período seco, seguido de um valor bem próximo em agosto, respectivamente de 1.272,7 kg.ha ${ }^{-1}$ e $1.236,1$ kg.ha- ${ }^{-1}$ e a menor deposição em junho, demonstrando características sazonais. A queda de folhas em florestas tropicais é, de modo geral, contínua, mas variável, apresentando um pico máximo na estação seca (GOLLEY, 1983; MEGURO et al., 1979). A ausência de extremos climáticos (períodos prolongados de frio intenso e seca), aliada à diversidade florística nessa região, permite que a floresta produza serapilheira durante todo o ano (Figura 1).

R. Árvore, Viçosa-MG, v.28, n.6, p.793-800, 2004 


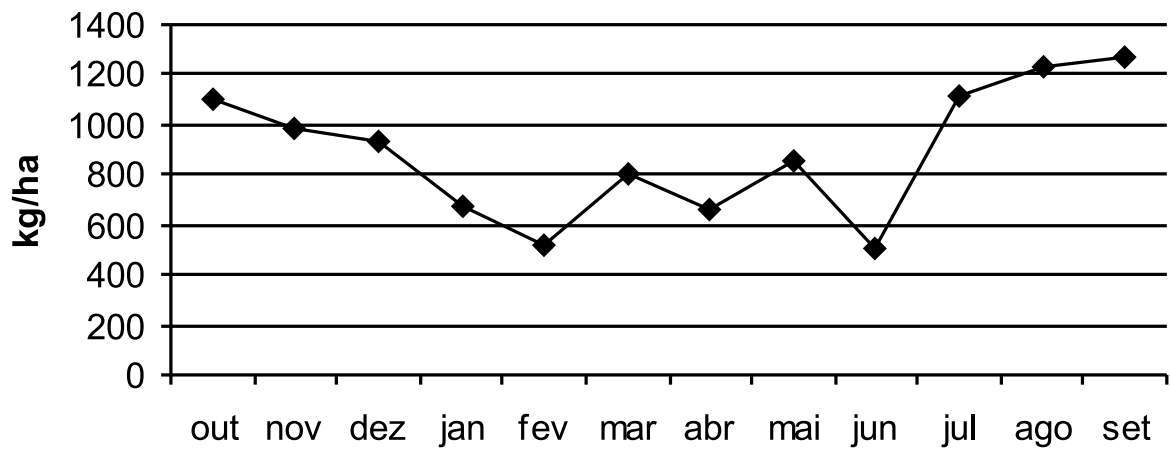

Figura 1 - Produção média mensal de serapilheira $\left(\mathrm{kg} \cdot \mathrm{ha}^{-1} \cdot \mathrm{a}^{-1}\right)$.

Figure 1 -Average monthly production of litter $\left(\mathrm{kg} \cdot \mathrm{ha} \mathrm{a}^{-1} \cdot \mathrm{y}^{-1}\right)$.

Os valores encontrados na produção anual de serapilheira situam-se dentro da faixa verificada nos ecossistemas florestais localizados no Estado de São Paulo(OLIVEIRA, 1997; PAGANO e DURIGAN, 2000).

\subsection{Conteúdo de macronutrientes na serapilheira}

No Quadro 1, mostram-se as concentrações médias de N, P, K, Ca e Mg na serapilheira derrubada.

Quadro 1 - Concentração média de macronutrientes na serapilheira, em 12 meses consecutivos de estudo (g.kg-1 de serapilheira)

Table 1 - Average concentration of litter macronutrients

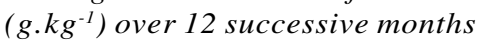

\begin{tabular}{lrrrrr}
\hline Mês & $\mathbf{N}$ & $\mathbf{P}$ & $\mathbf{K}$ & $\mathbf{C a}$ & $\mathbf{M g}$ \\
\cline { 2 - 6 } & \multicolumn{4}{c}{$\left(\mathrm{g} \cdot \mathrm{kg}^{-1} \mathrm{de}\right.$ serapilheira $)$} \\
\cline { 2 - 6 } Outubro/2000 & 21,00 & 1,20 & 11,00 & 20,00 & 6,00 \\
Novembro/2000 & 23,00 & 1,30 & 4,00 & 21,00 & 2,40 \\
Dezembro/2000 & 23,25 & 1,40 & 4,75 & 21,00 & 3,55 \\
Janeiro/2001 & 23,25 & 1,18 & 3,25 & 20,00 & 3,15 \\
Fevereiro/2001 & 21,75 & 1,45 & 7,25 & 21,25 & 3,80 \\
Março/2001 & 22,25 & 1,63 & 9,00 & 22,75 & 5,50 \\
Abril/2001 & 21,50 & 1,68 & 9,50 & 21,75 & 5,53 \\
Maio/2001 & 20,50 & 1,33 & 3,25 & 23,75 & 3,93 \\
Junho/2001 & 20,50 & 1,48 & 6,50 & 23,50 & 5,15 \\
Julho/2001 & 20,50 & 1,38 & 5,25 & 25,75 & 4,65 \\
Agosto/2001 & 24,00 & 1,23 & 5,00 & 28,00 & 4,98 \\
Setembro/2001 & 17,50 & 1,18 & 6,00 & 25,50 & 4,10 \\
\hline X & 21,58 & 1,37 & 6,23 & 22,85 & 4,40 \\
S & 1,76 & 0,17 & 2,52 & 2,52 & 1,09 \\
CV (\%) & 8,16 & 12,41 & 40,45 & 11,03 & 22,77 \\
\hline
\end{tabular}

R. Árvore, Viçosa-MG, v.28, n.6, p.793-800, 2004
O potássio e o magnésio foram os nutrientes que apresentaram maiores variações temporais em suas concentrações na serapilheira. Já as variações mensais dos conteúdos de $\mathrm{N}, \mathrm{P}$ e Ca foram relativamente pequenas.

Os meses com maior concentração de potássio na serapilheira foram aqueles em que a precipitação pluviométrica foi mais baixa, sendo as menores concentrações encontradas nos períodos de maior precipitação (Quadro 1). É possível ter havido deposição de fuligem de queimadas, ricas em potássio, oriundas da extensa área de cultivo de cana-de-açúcar presente na região.

Sarruge e Haag (1974) propuseram alguns padrões para as concentrações de fósforo, potássio, cálcio e magnésio em plantas: de 0,1 a $1,5 \%$ da matéria seca para o fósforo, estando as mais comuns entre $0,1 \mathrm{e}$ $0,3 \%$. As demais concentrações seriam de 1 a $5 \%$ de nitrogênio, 0,2 a $11 \%$ de o potássio, de 0,02 a $5 \%$ de cálcio e 0,02 a 2,5\% de magnésio. No presente estudo, as concentrações de fósforo, nitrogênio, potássio, cálcio e magnésio representam, respectivamente, $0,1 \%, 2,0 \%, 0,5 \%, 1,9 \%$ e $0,4 \%$, mostrando-se, portanto, dentro dos dados citados na literatura.

\subsection{Transferência de nutrientes através da serapilheira produzida e eficiência de utilização de nutrientes}

No Quadro 2, mostra-se a transferência anual de nutrientes pela queda de serapilheira na mata ciliar. 
Quadro 2-Transferência de N, P, K, Ca e Mg pela serapilheira $\left(\mathrm{kg} \cdot \mathrm{ha}^{-1} \cdot \mathrm{a}^{-1}\right)$, na área em estudo

Table $2-N, P, K, C a$ and $M g$ transfer by litter $\left(k g . h a^{-1} \cdot y^{-1}\right)$ in the target area

\begin{tabular}{|c|c|c|c|c|c|}
\hline \multirow[t]{2}{*}{ Mês } & $\mathbf{N}$ & $\mathbf{P}$ & $\mathbf{K}$ & $\mathbf{C a}$ & Mg \\
\hline & \multicolumn{5}{|c|}{ (g.kg ${ }^{-1}$ de serapilheira) } \\
\hline Outubro/2000 & 25 , & 1,45 & 13,31 & 24,24 & 7,26 \\
\hline $\mathrm{bro} / 2000$ & 22,32 & 26 & 8 & 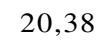 &, 32 \\
\hline D & 20 , & & & & 07 \\
\hline Jane & 10,2 & & & & ,44 \\
\hline $\mathrm{Fer}$ & & & & & , 00 \\
\hline & 14, & & 5,73 & 14 & 3,50 \\
\hline & & & 4, & & 2,43 \\
\hline & 14, & 7 & 2,38 & 17 & 2,87 \\
\hline & & & & & 1,31 \\
\hline & 25 & 1 & 1 & 31 , & 5,77 \\
\hline & 36 , & 1, & 7,64 & 42,78 & 7,60 \\
\hline Setembro/ & 28,34 & 0,03 & 0,01 & 0,19 & 0,13 \\
\hline Total & 217,76 & 11,55 & 52,79 & 199,80 & 38,70 \\
\hline$X$ & 18,1 & 0,96 & 4,40 & 16,65 & 3,23 \\
\hline $\mathrm{S}$ & & 0,57 & 3,59 & 12,10 & 2,43 \\
\hline CV $(\%)$ & 53,99 & 59,38 & 81,59 & 72,67 & 75,23 \\
\hline
\end{tabular}

O retorno total estimado de macronutrientes foi de 520,6 kg.ha-1 . O nitrogênio é o elemento que apresenta a maior transferência dentro da vegetação $(217,76$ $\left.\mathrm{kg} \cdot \mathrm{ha}^{-1}\right)$, seguido do Ca $\left(199,8 \mathrm{~kg} \cdot \mathrm{ha}^{-1} \cdot \mathrm{a}^{-1}\right)$. O retorno do cálcio é alto na maioria das florestas tropicais estudadas (VITOUSEK, 1984). Os demais macronutrientes eram constituídos $52,79 \mathrm{~kg} \cdot \mathrm{ha}^{-1}$ de potássio, $38,70 \mathrm{~kg} \cdot \mathrm{ha}^{-}$ ${ }^{1}$ de magnésio e $11,76 \mathrm{~kg}$.ha- ${ }^{-1}$ de fósforo, o qual se apresenta como elemento limitante no ecossistema. O retorno desses nutrientes, através da serapilheira, encontrase na seguinte ordem: $\mathrm{N}>\mathrm{Ca}>\mathrm{K}>\mathrm{Mg}>\mathrm{P}$.
Nas Figuras 2 e 3, mostram-se as transferências mensais de $\mathrm{N}, \mathrm{P}, \mathrm{K}, \mathrm{Ca}$ e $\mathrm{Mg}$ pela serapilheira na floresta estudada. A transferência de nutrientes ao solo acompanhou a tendência de deposição da serapilheira, como pode ser constatado nas Figuras 1, 2 e 3. Observase, nessas figuras, que o nitrogênio e o cálcio apresentam um valor de transferência mais elevado no final da estação seca (mês de agosto), com uma tendência de diminuição nos meses mais chuvosos (Figura 2). Para fósforo e potássio, as maiores taxas de transferência ocorreram no mês de outubro, com a chegada das primeiras chuvas (Figura 3). À exceção do Ca, os demais nutrientes apresentaram queda brusca com a chegada das primeiras chuvas, no mês de setembro (Figuras 2 e 3 ).

\subsection{Serapilheira acumulada e taxa de decomposição}

As coletas para a obtenção da estimativa da serapilheira acumulada e da taxa de decomposição foram bimensais. A média anual de serapilheira acumulada foi de $6.227,3 \mathrm{~kg} \cdot \mathrm{ha}^{-1}$, e a taxa instantânea de decomposição da serapilheira $(\mathrm{K})$, que indiretamente representa a velocidade com que os nutrientes, ligados a ela, tornamse disponíveis, foi de 1,71. Esse valor é considerado alto, segundo o critério de Olson (1963). Pagano (1989) considerou alto o valor de 1,15 para a constante $K$, porém a literatura cita valores ainda mais elevados (Quadro 3).

O estoque médio estimado de serapilheira acumulada foi inferior ao relatado por Cunha et al. (1993) (6,7 t.ha $\left.{ }^{-1}\right)$ e Oliveira (1997) $(7.745,43$ kg.ha-1 $)$. Porém, inserese dentro do intervalo observado em cinco Florestas Semidecíduas no Sudeste do Brasil, onde foram encontrados valores de serapilheira acumulada entre 5,5 e 8,6 t.ha' ${ }^{-1}$ (MORELLATO, 1992).

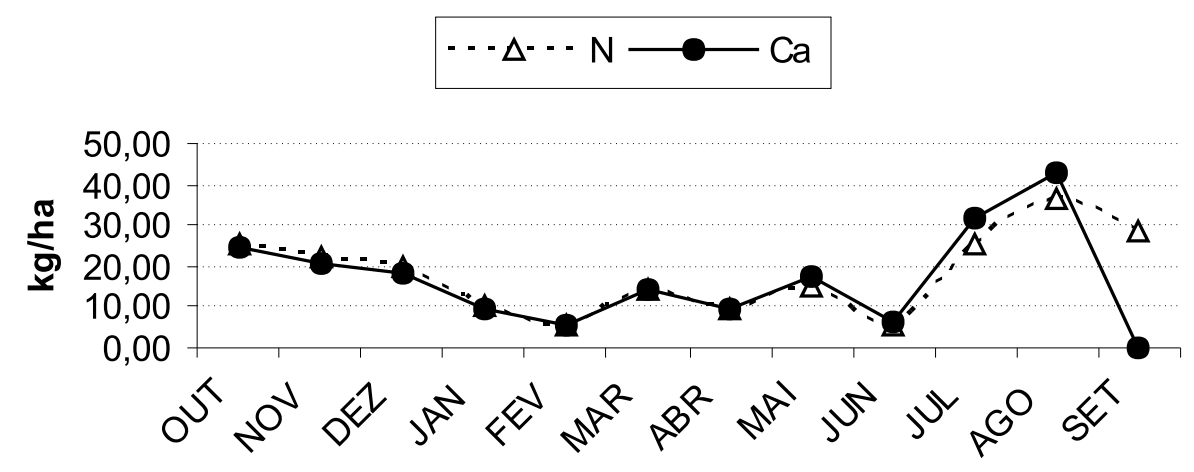

Figura 2 - Total de $\mathrm{N}$ e Ca transferidos pela serapilheira.

Figure 2 - Total $N$ and Ca transferred by litter. 


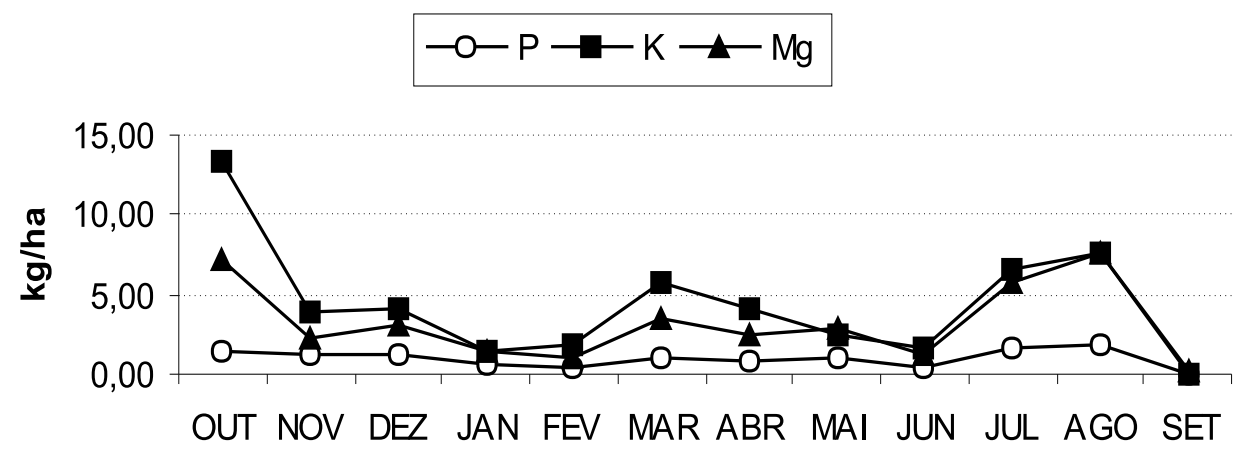

Figura 3 - Total de $\mathrm{P}, \mathrm{K}$ e $\mathrm{Mg}$ transferidos pela serapilheira.

Figure 3 - Total $P$, K and $M g$ transferred by litter.

Quadro 3 - Valores de K (constante de deposição) em ecossistemas florestais Table 3 - K values (decomposition constant) for forest ecosystems

\begin{tabular}{lcl}
\hline Tipo de Floresta & K & Fonte \\
\hline Floresta Estacional Semidecidual & 1,2 & Cunha, 1997 \\
Floresta Estacional Semidecidual & 1,9 & Oliveira, 1997 \\
Floresta Estacional Semidecidual & 1,6 & Morellato, 1992 \\
Floresta Estacional Decidual & 1,2 & Cunha et al., 1993 \\
Floresta Estacional Semidecidual & 1,7 & Presente estudo \\
\hline
\end{tabular}

Os valores calculados para o tempo médio de renovação da quantidade de serapilheira acumulada sobre o solo e o tempo necessário para o desaparecimento de 50 e $95 \%$ da serapilheira, segundo a metodologia de Shanks e Olson (1961), encontramse no Quadro 4.
O tempo necessário para o desaparecimento de 50 e $95 \%$ da serapilheira foi, respectivamente, de 150 e 639 dias, confirmando uma taxa de decomposição muito rápida na área estudada, o que indica um rápido reaproveitamento de nutrientes por parte da vegetação (PAGANO, 1989).

Quadro 4 - Taxa de decomposição (K), tempo médio de renovação (1/K) e tempo necessário para decomposição de 50 e $95 \%$ da serapilheira

Table 4-Decomposition rate (K), average time of renovation $(1 / K)$ and necessary time for 50 and at $95 \%$ litter decomposition

\begin{tabular}{cccc}
\hline Coeficiente de Decomposição $(\mathrm{K})$ & $1 / \mathrm{K}($ anos $)$ & T $0,5($ anos $)$ & T $0,05($ anos $)$ \\
\hline 1,71 & 0,58 & 0,41 & 1,75 \\
\hline
\end{tabular}

\section{CONCLUSÃO}

A metodologia empregada para estudar a produção de serapilheira e a ciclagem de nutrientes foi satisfatória e propiciou uma boa estimativa para o total de serapilheira produzida, a qual alcançou um valor máximo de deposição de biomassa no fim do período seco, demonstrando características sazonais, com pequena variação nas concentrações dos nutrientes, no decorrer do ano, e evidenciando que o fornecimento destes ao solo ocorre constantemente. Observou-se pouco estoque de serapilheira acumulada na superfície do solo, apresentando uma rápida velocidade de decomposição

R. Árvore, Viçosa-MG, v.28, n.6, p.793-800, 2004 
e, conseqüentemente, rápido aproveitamento de nutrientes por parte da vegetação, o que favorece a ciclagem e o equilíbrio desse ecossistema. Os resultados indicaram o importante e significativo papel desenvolvido pelas matas ciliares na ciclagem de nutrientes.

\section{AGRADECIMENTOS}

À FAPESP, pelo financiamento concedido, Processo ${ }^{\circ}$ 99/12415-1.

\section{REFERÊNCIAS BIBLIOGRÁFICAS}

BRAY, J.R.; GHORAN, E. Litter production in forest of the world. Advances Ecology of Research, v.2, p.101-157, 1964.

CARVALHO, W.; PANOSO, L.A.; MORAES, M.H. Levantamento semidetalhado dos solos da Fazenda Experimental Edgardia, Município de Botucatu - SP. Botucatu: Universidade de São Paulo, 1991. (Boletim Científico da Faculdade Ciência Agronômica, 2).

CUNHA, G.C. Aspectos da ciclagem de nutrientes em diferentes fases sucessionais de uma floresta estacional do Rio Grande do Sul. 1997. 86f. Dissertação (Mestrado em Ciências/Ciências Florestais) - Escola Superior de Agricultura "Luiz de Queiroz", Piracicaba, 1997.

CUNHA, G.C. et al. Dinâmica nutricional em floresta estacional decidual com ênfase aos minerais provenientes da deposição da serapilheira. Ciência Florestal, v. 3, n.1, p.35-64, 1993.

DELITTI, W.B.C. Ciclagem de nutrientes minerais em matas ciliares. In: SIMPÓSIO SOBRE MATA CILIAR, 1989, São Paulo. Anais... São Paulo: Fundação Cargil, Secretaria do Meio Ambiente, Instituto de Botânica, 1989. p.88-98.

EMPRESA BRASILEIRA DE PESQUISA AGROPECUÁRIA - EMBRAPA. Centro Nacional de Pesquisa de Solos. Sistema brasileiro de classificação de solos. Brasília: 1999. 412p.

GOLLEY, F.B. Tropical rain forest ecosystems: structure and function. Amsterdam: Elsevier, 1983. 392p.
INSTITUTO BRASILEIRO DE GEOGRAFIA E ESTATÍSTICA - IBGE. Manual técnico da vegetação brasileira. Rio de Janeiro: 1992. $123 \mathrm{p}$.

MEGURO, M.; VINUIZA, G.N.; DELITTI, W.B.C. Ciclagem de nutrientes na mata mesófila secundária I - Produção e conteúdo de nutrientes minerais no folhedo. Boletim de Botânica da Universidade de São Paulo, n.7, p.1131, 1979.

MORELLATO, L.P.C. Nutrient cycling in two south-east brazilian forests I- Litterfall and litter standing crop. Journal Tropical Ecology, v. 8, p.205-205, 1992.

OLIVEIRA, R.E. Aspectos da dinâmica de um fragmento florestal em Piracicaba - SP: silvigênese e ciclagem de nutrientes. 1997. 79f. Dissertação (Mestrado em Ciências/ Ciências Florestais) - Escola Superior de Agricultura “Luiz de Queiroz”, Piracicaba, 1997.

OLSON, J.S. Energy storage and the balance of producers in ecological systems. Ecology, v.44, p.322-331, 1963.

PAGANO, S.N. Nutrientes minerais no folhedo produzido em mata mesófila semidecídua no município de Rio Claro, SP. Revista

Brasileira de Biologia, v. 49, p.641-647, 1989.

PAGANO, S.N.; DURIGAN, G. Aspectos da ciclagem de nutrientes em matas ciliares do Oeste do Estado de São Paulo, Brasil. In: RODRIGUES, R.R.; LEITÃO FILHO, H.F. (Eds.). Matas ciliares: conservação e recuperação. São Paulo: EDUSP/FAPESP, 2000. p.109-123.

POGGIANI, F.; SCHUMACHER, M.V. Ciclagem de nutrientes em florestas nativas. In: GONÇALVES, J.L.M.; BENEDETTI, V. (Eds.). Nutrição e fertilização florestal. Piracicaba: IPEF, 2000. 427p.

SARRUGE, J.L.; HAAG, H.P. Análise química em plantas. Piracicaba: Escola Superior de Agricultura Luiz de Queiroz, 1974. 56p.

R. Árvore, Viçosa-MG, v.28, n.6, p.793-800, 2004 
SCHUMACHER. M.V. Aspectos da ciclagem de nutrientes e do microclima em talhões de Eucalyptus camadulensis Dehnh, Eucalyptus grandis Hill ex Maiden e Eucalyptus torelliana F. Muell. 1992. $87 \mathrm{f}$.

Dissertação (Mestrado em Ciências/Ciências Florestais) - Escola Superior de Agricultura "Luiz de Queiroz", Piracicaba, 1992.

SHANKS, R.; OLSON, J.S. First year breakdown of leaf litter in Southern Appalachia Forest Science, v.134, p.194-195, 1961.
VITOUSEK, P.M. Litterfall, nutrient cycling and nutrients in tropical forests. Ecology, v.65, n.1, p.285-298, 1984.

VITOUSEK, P.M. Nutrient cycling and nutrient use efficiency. American Naturalist, v.119, n.4, p.553-72, 1982.

WARING, R.H.; SCHLESINGER, W.H. Decomposition and forest soil development. In: FOREST ecosystems: concept and management. New York: Academic Press, 1985. 340p. 